Rev Biomed 2006; 17:3-9.

\title{
Daño fetal en la descendencia de mujeres heterocigóticas para las hiperfenilalaninemias.
}

Artículo Original

Zoe Robaina-Jiménez, Bárbara Barrios-García, Enna Gutiérrez-García, Laritza Martínez-Rey, Víctor Tamayo-Chang, Águeda N. Pereira-Pérez.

Centro Nacional de Genética Médica CUBA, Centro colaborador de la OMS para el desarrollo de los enfoques genéticos en la promoción de salud. Centro Provincial de Genética de Holguín. Cuba.

\section{RESUMEN.}

Introducción. En mujeres heterocigóticas para el gen fenilalanina hidroxilasa, ante una sobrecarga dietética durante el embarazo, se pueden encontrar cifras elevadas de fenilalanina que pueden conducir a diversos trastornos en el desarrollo fetal, fundamentalmente a nivel del Sistema Nervioso Central.

Objetivos. Determinar si existe asociación entre el estado heterocigótico materno para las hiperfenilalaninemias y el daño fetal. Caracterizar clínicamente a los hijos de las mujeres heterocigóticas.

Material y Método. Se estudiaron 34 mujeres procedentes de familias de fenilcetonúricos y con hiperfenilalaninemia persistente de las provincias de Villa Clara, Cienfuegos y Holguín. Se utilizó la prueba de tolerancia a la fenilalanina, determinando la condición de heterocigóticas para alguna mutación de los genes que participan en el metabolismo de la fenilalanina y se relacionó con la presencia de anomalías específicas en la descendencia de las mismas.

Resultados. El retraso mental fue el efecto de daño fetal más frecuente, tanto la descendencia de heterocigóticas, como la de hiperfenilalaninemicas resultó afectada. En los hijos de madres hiperfenilalaninemicas se observó un predominio de las convulsiones (asociado a retraso mental) y del retraso mental aislado. Sin embargo, en la descendencia de madres heterocigóticas, el retraso mental constituye la afección más importante.

Conclusiones. Se evidencia la presencian de alteraciones del SNC y otros en la descendencia de mujeres heterocigóticas para las hiperfenilalaninemias, lo cual incentiva a continuar profundizando en esta asociación y se recomienda profundizar en la relación fenotipo-genotipo.

(Rev Biomed 2006; 17:3-9)

Palabras clave: efecto teratogénico, fenilcetonuria, hiperfenilalainemias, retraso mental.

\section{SUMMARY.}

Fetal damage in offspring of heterozygous women for hyperphenylalaninemias.

Background. It is possible to find high plasmatic phenyalanine concentration in heterozygous women for the phenylalanine hydroxylase gene, when there

Solicitud de sobretiros: Dra. Zoe Robaina-Jiménez. Capitán Velazco \# 22 entre Cuba y Colón, Santa Clara, Villa Clara, Cuba. Correo electrónico: zoe@giron.sld.cu

Recibido el 28/Julio/2005. Aceptado para publicación el 8/Febrero/2006.

Este artículo está disponible en http://www.uady.mx/sitios/biomedic/revbiomed/pdf/rb061712.pdf

Vol. 17/No. 1/Enero-Marzo, 2006 


\section{Z Robaina-Jiménez, B Barrios-García, E Gutiérrez-García, L Martínez-Rey y col.}

is a phenylalanine intake during pregnancy, and it can lead to disorders in the foetus, specifically in the foetal central nervous system (CNS).

Objective. To determine if there is any association relation between the maternal heterozygotic state for the hyperphenylalanine gene and the appearance of foetal disorders. To perform a clinic characterization of those heterozygotic women's offspring.

Design. 34 women from phenylketonuria and hyperphenylalaninemia families from Villa Clara, Cienfuegos and Holguín were studied. The phenylalanine tolerance test was used to determine the condition of heterozygotic women for any mutation of the gene involved in the phenylalanine metabolism, and to relate it to the existing specific disorders in their offspring.

Results. Mental retardation was the most frequent clinical finding. The offspring of heterozygotic and hyperphenylalaninemia women were both affected. In the children of hyperphenylalaninemic mothers convulsions (associated to mental retardation) as well as mental retardation were the predominant findings, and isolated mental retardation was found predominantly in the offspring of heterozygotic mothers.

Conclusions. There is evidence of association between the CNS disorders and others clinical findings in the offspring and the maternal state of heterozygotic in hyperphenylalaninemia. We recommend carrying our a subsequent study to determine the phenotype-genotype relationship.

(Rev Biomed 2006; 17:3-9)

Key words: teratogenic damage, phenylketonuria, hyperphenylalaninaemia, heterocygotic, mental retardation.

\section{INTRODUCCIÓN.}

Los errores innatos del metabolismo son enfermedades monogénicas, de herencia autosómica recesiva en su mayoría. La alteración de un gen produce un defecto enzimático, que conduce a las alteraciones bioquímicas características de cada enfermedad metabólica y que son responsables de los fenotipos desadaptativos según cada patología (1).

La fenilcetonuria (PKU) es una alteración metabólica que se debe a una deficiencia para metabolizar el aminoácido fenilalanina, el cual se acumula en sangre y afecta múltiples tejidos, incluyendo el sistema nervioso central, ocasionando fundamentalmente retraso mental (RM). Para prevenir las anormalidades clínicas es necesario el diagnóstico precoz y la aplicación temprana de una dieta pobre en fenilalanina sólo con la cantidad suficiente de este aminoácido para el crecimiento normal del niño (2).

La PKU es un ejemplo por excelencia de efecto materno teratogénico sobre el desarrollo del feto. Varias publicaciones se han realizado, pero muchas preguntas han quedado sin respuestas. Por Ejemplo es sabido que el efecto teratogénico de la fenilalanina en las mujeres fenilcetonúricas e hiperfenilalaninémicas se debe a cifras elevadas de fenilalanina en suero fetal, debido a la existencia de un gradiente transplacentario positivo para este aminoácido (aa) (4). Independientemente de que, en condiciones normales las cifras séricas de fenilalanina en mujeres heterocigóticas suelen estar por debajo de $120 \mu \mathrm{mol} / \mathrm{L}$, luego de una sobrecarga de alimentos ricos en proteínas, estas mujeres metabolizan lentamente estos productos, pudiendo mantener durante varias horas cifras elevadas de este aa en suero, que por el conocido efecto de bomba pueden llegar al feto en concentraciones teratogénicas durante este periodo, que pudiera ocasionar un daño de menor o mayor magnitud. Por esta razón se decidió estudiar la asociación existente entre el estado heterocigótico de las madres y los daños fetales, que nos ayudara estimar la posible eficacia de una dieta restrictiva en fenilalanina preconcepcionalmente y durante la gestación con el objetivo de garantizar el desarrollo adecuado del SNC de sus hijos. Por lo que debemos preocuparnos por la repercussion que pueda tener esta condición y su posible efecto

\section{Revista Biomédica}




\section{Daño fetal en la hiperfenilalaninemia de heterocigóticas.}

teratogénico.

El estricto control bioquímico antes de la concepción y durante el embarazo es universalmente reconocido, específicamente se recomienda una concentración menor que $5 \mathrm{mg} / \mathrm{dL}$ de fenilalanina en sangre. Asimismo, consideramos que existen algunos aspectos que necesitan ser aclarados (3).

\section{PACIENTES Y MÉTODOS.}

Se realizó un estudio descriptivo de corte transversal, donde la población estudiada fue de 16 familias de pacientes afectados con fenilcetonuria o con hiperfenilalaninemia persistente y la muestra 34 mujeres y 39 de sus hijos ambos grupos, familiares de pacientes fenilcetonúricos o con hiperfenilalaninemia persistente

Se procedió a la búsqueda de mujeres familiares de los individuos que presentaban la condición de fenilcetonúricos e hiperfenilalaninemia persistente al nacimiento en 1989 en la provincia de Villa Clara, Cienfuegos y Holguín de los registrados hasta el año 2000 en el Centro Nacional de Genética Medica de Cuba, con la ayuda de los centros provinciales se procedió a la localización domiciliaria. Utilizando como criterio de inclusión aquellos que desearan participar en el estudio, a través de la firma del consentimiento informado, familiares de los pacientes fenilcetonúricos y con hiperfenilalaninemia persistente, mujeres y sus hijos pertenecientes a estas familias residentes en las provincias en estudio, excluyendo los hijos afectado por un daño de causa conocida y que tengan menos de 3 años.

A estas mujeres incluidas en el estudio se les extrajo $5 \mathrm{ml}$ de sangre en ayunas, luego se les administraron $100 \mathrm{mg} / \mathrm{Kg}$. de fenilalanina acompañado con algún jugo de cítrico, se les extrajo
$5 \mathrm{~mL}$ de sangre total a la hora de administrado el preparado, a las 2 y a las 3 horas. Las muestras se centrifugaron y se conservaron en refrigeración no más de 48 horas. Luego se cuantificó fluorimétricamente la fenilalanina.

A cada muestra se le determinó la concentración de fenilalanina en suero y se compararon con los valores normales (5), además se conservaron muestras de ADN para futuras investigaciones de mutaciones.

Posteriormente se realizó un interrogatorio a los padres de los niños en estudio. Los datos de la encuesta fueron registrados en un modelo confeccionado al efecto, así como el examen físico efectuado, que incluyó: circunferencia cefálica, alteraciones físicas y motoras y se evaluó el coeficiente de inteligencia a través de pruebas psicométricas.

\section{RESULTADOS.}

El desarrollo de nuestra investigación permitió estudiar a 34 madres, a las cuales se le realizó la prueba de tolerancia a la fenilalanina con las curvas correspondientes para cada condición y de éstas, la mayoría resultaron ser portadoras para una mutación en el gen de la PAH (cuadro 1, figura 1).

En los hijos de las madres estudiadas el retraso mental fue el mayor efecto de daño fetal, seguido por las disfunciones cerebrales mínimas. Al realizar una distribución por grupos según el grado de retraso mental, se encontró un predominio para el retraso mental ligero y severo y un número menor de casos para el retraso mental moderado.

En el cuadro 2 se puede apreciar que tanto los descendientes de las portadoras como los de madres hiperfenilalaninemicas presentaron alguna manifestación (daño), donde es válido señalar, que

\section{Cuadro 1}

Distribución de mujeres analizadas en nuestro estudio según el resultado de la prueba de fenilalanina.

\begin{tabular}{cc|lc|cc|cc}
\hline \multicolumn{2}{c|}{ Normales } & \multicolumn{2}{c|}{ Heterocigótica } & \multicolumn{2}{|c|}{ Hiperfenilalaninemia } & \multicolumn{2}{|c}{ Total } \\
\hline No. & $\%$ & No. & $\%$ & No. & $\%$ & No. & $\%$ \\
8 & 23.53 & 22 & 64.71 & 4 & 11.76 & 34 & 100 \\
\hline
\end{tabular}


Z Robaina-Jiménez, B Barrios-García, E Gutiérrez-García, L Martínez-Rey y col.

\section{Cuadro 2}

Relación entre la condición de la madre y la existencia de daño fetal.

\begin{tabular}{l|cc|cc|cc}
\hline \multirow{2}{*}{$\begin{array}{l}\text { Condición } \\
\text { materna }\end{array}$} & \multicolumn{2}{|c}{ Si } & \multicolumn{2}{c}{ No } & \multicolumn{2}{c}{ Total } \\
& No. & $\%$ & No. & $\%$ & No. & $\%$ \\
\hline Normales & 0 & 0 & 8 & 100 & 8 & 100 \\
Heterocigótica & 19 & 86.36 & 3 & 13.63 & 22 & 100 \\
\hline HPA & 4 & 100 & 0 & 0 & 4 & 100 \\
\hline
\end{tabular}

se observa una relación positiva entre la condición de heterocigótica y la frecuencia del daño fetal, que sugiere una asociación entre ellas. Al caracterizar la descendencia de las madres HPA, se observó como manifestación más frecuente las convulsiones (asociadas a retraso mental) y el retraso mental aislado (figura1). Sin embargo, en la descendencia de madres heterocigóticas, el retraso mental constituye la afección más importante y a diferencia del grupo anterior no aparecen casos de microcefalia (cuadro 3).

\section{DISCUSIÓN.}

En la muestra estudiada encontramos 22 mujeres portadoras, puede afirmarse que estos resultados se deben al método de selección de la muestra, ya que se incluyeron para nuestro estudio aquellas mujeres familiares de pacientes fenilcetonúricos y con hiperfenilalaninemia persistente, donde con mayor probabilidad existe

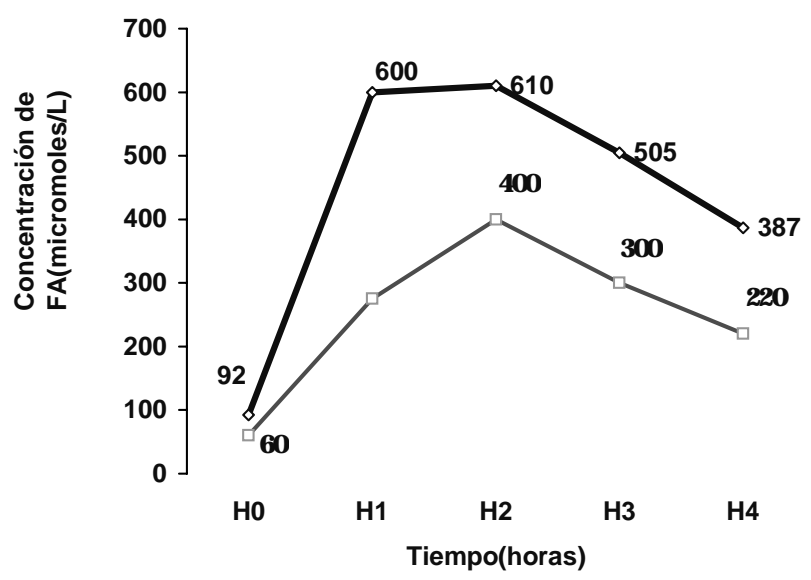

Figura 1.- Curva de degradación de fenilalamina en suero de sujetos normales ( $\square$ ) y portadoras ( $\square \longrightarrow$ ). Revista Biomédica un número incrementado de portadoras, por ser precisamente en estas familias donde se está segregando el gen deletéreo.

El incremento de la fenilalanina en sangre materna tiene un mayor efecto a nivel del sistema nervioso central que en el resto del organismo fetal. Este hecho puede estar relacionado con los diferentes grados de elevación de la concentración de fenilalanina para la ocurrencia de un fenotipo más severo en la descendencia, unido a otros factores, como la susceptibilidad individual, momento del embarazo en que ocurrió esta elevación, así como el genotipo materno específico y la falta de control dietético restrictivo, para la fenilalanina, durante el embarazo.

Podemos considerar el retraso mental como un daño que pudiera estar relacionado con este efecto teratogénico, ya que el retraso mental en Cuba, tiene una prevalecía de 1,25\% (6), según estudios recientes y en la muestra estudiada se alcanzó un valor tan alto como el 50\%, lo que resulta significativo. Sin embargo, al tener en cuenta el reducido tamaño de la muestra, consideramos importante la realización de estudios futuros, con un mayor número de pacientes.

\section{Cuadro 3}

Caracterización clínica de los hijos de madres Hiperfenilalaninemicas y Portadoras.

Manifestación Clínicas Hiperfenil (n=4) Portadoras (n=22)

\begin{tabular}{lcc}
\hline Retraso mental & $2(50 \%)$ & $10(45 \%)$ \\
Microcefalia & $1(25 \%)$ & 0 \\
Convulsiones & $3(75 \%)$ & $4(18 \%)$ \\
Hiperquinesia & $1(25 \%)$ & $1(4.5 \%)$ \\
\hline
\end{tabular}




\section{Daño fetal en la hiperfenilalaninemia de heterocigóticas.}

Por considerar que las mujeres potadoras, al exponerse a una sobrecarga de fenilalanina durante el embarazo, similar a la que se provocó con la prueba de tolerancia, pudieran desarrollar un fenotipo metabólico similar a las mujeres fenilcetonúricas e hiperfenilalaninémicas, con las posibles consecuencias para su descendencia, se valoraron los resultados de la bibliografía encontrada para estas condiciones.

En los Estados Unidos, en el período de 19982000, se realizó un estudio de seguimiento del control dietario de embarazadas con fenilcetonuria y sus resultados demostraron que estas embarazadas, cuando no tenían un control dietario antes y durante el embarazo, su descendencia tenía un $93 \%$ de riesgo para retraso mental y $72 \%$ de riesgo para microcefalia. Estos riesgos resultaron del efecto tóxico de los altos niveles sanguíneos de fenilalanina durante el embarazo y no porque la descendencia padeciera de fenilcetonuria (7-12). Similar a lo encontrado en el presente estudio, donde predominó el retraso mental o algún daño al SNC.

Existen muchas propuestas de mecanismos responsables del daño neurológico a los hijos, por lo que queda mucho por aclarar. Algunos autores refieren varias condiciones que proponen la explicación del desarrollo anormal del cerebro, como los errores innatos del metabolismo en la producción de tóxicos o deficiente energía intrauterina, modificación del contenido y función de membranas o trastornos en la expresión normal de genes intrauterinos responsables de la morfogénesis (13-15).

Otros investigadores proponen un trastorno en el metabolismo de los ácidos grasos polinsaturados como factor etiológico en los hijos de madres fenilcetonuricas, sugiriendo a éstos como responsables de la microcefalia y el retraso mental (16).

Al profundizar en las bases bioquímicas de la PAH, constataron que el ácido fenilpirúvico (uno de los productos intermediarios de la vía metabólica de la hidroxilación de la fenilalanina) era un inhibidor de la enzima piruvato carboxilasa en el cerebro, pero no en el hígado. Esto sugirió ser la causa de un defecto de mielinización del sistema nervioso central de estos pacientes (en ellos las concentraciones de ácido fenilpirúvico están aumentadas, debido a la afectación que sufre esta vía metabólica), así como de la presencia del retraso mental que los caracteriza, fundamentalmente en los casos de fenilcetonuria (17).

Se examinó el efecto de la hiperfenilalaninemia materna en el desarrollo morfológico y bioquímico del cerebro y el cerebelo de ratas. En este modelo fue evaluado un número de marcadores de estrés oxidativo y demostraron que la fenilcetonuria incrementa fuertemente los marcadores de estrés oxidativo estudiados e induce un daño morfológico significativo, concluyendo que este estado involucra un daño del cerebro y del cuerpo (18).

Algunos autores han estudiado la posible relación entre ciertos genotipos maternos y el fenotipo de la descendencia y observaron como varias mutaciones que producen proteínas truncadas, largas deleciones y alteraciones en el sitio activo de la enzima, generalmente están relacionadas con los fenotipos más severos, aunque efectos menos severos son posibles (19-21).

A pesar de la limitación debida a la gran heterogeneidad de mutaciones, se puede arribar a una conclusión general, cuando la mutación es más severa. Los resultados sugieren que el rastreo de las variaciones de los niveles de tirosina plasmática pueden ser más sensibles que los de la fenilalanina plasmática (22).

También la susceptibilidad individual juega un papel importante en la variabilidad fenotípica según lo explican Pey y col., al referir que las variaciones metabólicas pueden ser explicadas por diferencias interindividuales, dada la inconsistencia para algunas mutaciones que se observan en pacientes con similares genotipos y diferentes fenotipos (23).

Si en las HPA y las fenilcetonúricas el genotipo materno, incluyendo el tipo de mutaciones, determinaría la funcionabilidad de la enzima y su efecto en la descendencia, además de la 


\section{Z Robaina-Jiménez, B Barrios-García, E Gutiérrez-García, L Martínez-Rey y col.}

susceptibilidad individual en las heterocigóticas, pudiera existir una relación similar.

Es notable que la frecuencia de microcefalia para hijos de madres HPA es muy elevada, en contraste con la frecuencia de este defecto para la población en general (2.5\%)(7). Es importante valorar qué tiempo este aminoácido se mantiene elevado en sangre y en qué niveles, lo cual puede observarse mediante la curva obtenida en la prueba de tolerancia a la fenilalanina, ya que sería muy sugestivo pensar que, si este incremento se mantiene, el efecto en la descendencia pudiera ser mayor de lo esperado

Se ha manifestado la susceptibilidad al daño por la elevación de este aminoácido, explicando que la frecuencia de las anomalías presentes en los hijos de madres fenilcetonuricas es proporcional a las concentraciones sanguíneas de fenilalanina materna. Si una dieta restrictiva de proteína es seguida durante y antes de la concepción, el riesgo de anomalías no es más alto que en la población normal (22-24). Los niveles de fenilalanina deben ser mantenidos entre 120 y $250 \mu \mathrm{mol} / \mathrm{L}$ y niveles de tirosina entre 45 y $90 \mu \mathrm{mol} / \mathrm{L}(22,25)$. Otros autores recomiendan que los niveles de fenilalanina sanguínea durante el embarazo deben ser entre 120 y $360 \mu \mathrm{mol} / \mathrm{L}$ (26-28). Los niveles elevados de fenilalanina materna durante el embarazo, como efecto teratogénico, puede resultar en un retardo del crecimiento, significativos trastornos psicomotores y defectos al nacimiento en hijos de embarazadas no tratadas ni sometidas a seguimiento, incluyendo todas las formas y variantes más ligeras de hiperfenilalaninemia, las que deben recibir el asesoramiento concerniente al riesgo de tener efectos fetales adversos $(9,29,30)$.

Concentraciones más elevadas se relacionan con afecciones cardíacas. La frecuencia y los tipos de enfermedades cardíacas congénitas en la descendencia de embarazos de mujeres con hiperfenilalaninemia, la coartación de la aorta y el síndrome de cavidades izquierdas está sobrerrepresentada. Valores mayores a $900 \mu \mathrm{mol} /$ $\mathrm{L}$ de fenilalanina materna incrementarían el riesgo para sus hijos $(31,32)$ pueden incrementar el riesgo para las anomalías cardíacas congénitas.

La prevención de estas enfermedades requiere la iniciación de una dieta baja en fenilalanina antes de la concepción o tempranamente en el embarazo. Sería recomendable la adopción de dicha dieta por las heterocigóticas embarazadas.

\section{REFERENCIAS.}

1.- Raimann BE, Cornejo EV. Una primera aproximación al diagnóstico y tratamiento de errores innatos del metabolismo. En: Colombo CM, Cornejo EV, Raimann BE, editoras. Errores innatos en el metabolismo del niño. 1a ed. Santiago de Chile: Editorial Universitaria; 1999. p. 4556.

2.- Campistol J, Vilaseca MA, Cambra FJ, Lambruschini N. Diagnóstico, tratamiento y seguimiento de las hiperfenilalaninemias. Act Nutr 1998; 24: 22-9.

3.- Walter JH. Inborn errors of metabolism and pregnacy. J Inherit Metab Dis 2000; 23: 229-36.

4.- Scott T' Fife, Hart D. Maternal phenylketonuria Abnormal baby despite low phenilalanine diet during pregnancy. Arch Dis Child 1980; 55: 634-47.

5.- Gutiérrez E, Barrios B, Mar J, Echevarría P, Damiani A, Alonso F. Estudio de portadores de Fenilcetonuria y otras Hiperfenilalaninemias por prueba de tolerancia a la fenilalanina. Rev Cubana Pediatr 1993; 65:88-92.

6.- Colectivo de autores. Por la vida. Casa Editorial Abril. Ciudad de la Habana.2003; 56-62.

7.- Barriers. To dietary control among pregnant women with phenylketonuria--United States, 1998-2000. MMWR 2002; 51:117-20.

8.- American Academy of Pediatrics: Maternal phenylketonuria. Pediatrics 2001; 107: 427-8.

9.- Moslinger D, Scheibenreiter S, Spoula E StocklerIpsiroglu S. Maternal phenylketonuria: dietary treatment of 2-generation illness. Geburtsh Neonatol 2000; 204 :1816.

10.- Levy HL, Guldberg P, Guttler F, Hanley WB, Matalon $\mathrm{R}$, Rouse $\mathrm{BM}$, et al Congenital heart disease in maternal phenylketonuria: report from the Maternal. Pediatr Res 


\section{Daño fetal en la hiperfenilalaninemia de heterocigóticas.}

2001; 49: 636-42.

11.- Sweeney E, Fryer A. Nasomaxillary hypoplasia and severe orofacial clefting in a child of a mother with phenylketonuria. J Inherit Metab Dis 2002; 25:77-9.

12.- Zaffanello M, Zamboni G, Tato L. Growth parameters in newborns with hyperphenylalaninaemia. Paediatr Perinat Ep 2002; 16:274-7.

13.- Nissenkorn A, Michelson M, Ben-Zeev B, LermanSagie T. Inborn errors of metabolism: a cause of abnormal brain development. Neurology 2001; 56:1265-72.

14.- Gressens P, Mesples B, Sahir N, Marret S, Sola A. Environmental factors and disturbances of brain development. Semin Neonatol 2001; 6:185-94.

15.- Oberdoerster J, Guizzetti M, Costa LG. Effect of phenylalanine and its metabolites on the proliferation and viability of neuronal and astroglial cells: possible relevance in maternal phenylketonuria. J Pharmacol Exp Ther 2000; 295:295-301.

16.- Infante JP, Huszagh VA. mpaired arachidonic (20:4n6) and docosahexaenoic (22:6n-3) acid synthesis by phenylalanine metabolites as etiological factors in the neuropathology of phenylketonuria. Mol Genet Metab 2001; 72:185-98.

17.- Arcas G. Las hiperfenilalaninemias persistente y el estado heterocigótica para mutaciones del gen fenilalnina hidroxilasa como factor de daño fetal. Tesis para optar por el título de especialista de I grado en genética clínica. ICBP "Victoria de Girón", Ciudad de la Habana. Diciembre, 2000.

18.- Martinez-Cruz F, Pozo D, Osuna C, Espinar A, Marchante C, Guerrero J.M. Oxidative stress induced by phenylketonuria in the rat: Prevention by melatonin, vitamin E, and vitamin C. J Neurosci Res 2002; 69: 550-8.

19.- Jennings IG, Cotton R, Kobe B. Structural interpretation of mutations in pheniylalanine hydroxylase protein aids in identifying genotype- phenotype correlations in phenylketonuria. Eur J Hum Genet 2000; 8: 683-96.

20.- Waters PJ. How PHA gene mutations cause hyperphenylalaninemia and mechanism matters: insights from invitro expression. Hum Mutat. 2003; 21: 357-69.

21.- Waters PJ, Parniak MA, Akeman BR, Scriver CR.
Characterization of phenylketonuria missense substitutions, distant from the phenylalanine hydroxylase active site illustrates a paradigm for mechanism and potential modulation of phenotype. Mol Genet Metab $2000 ; 69: 101-10$.

22.- Verduci E, Riva E, Agostoni C, Leviti S, Fiori L, Lammardo AM, et al. Phenylalanine hydroxylase mutations and phenylalanine-tyrosine metabolism in heterozygote for phenylalanine hydroxylase deficiency. Acta Paediatr 2002; 91: 805-10.

23.- Pey AL, Desviat LR, Gómez A, Ugarte M, Pérez B. Phenylketonuria genotype phenotype correlations based on expression analysis of structural and funtional mutations in PAH. Hum Mutat 2003; 21:370 -8.

24.- Purnell H. Phenylketonuria and maternal phenylketonuria. Breastfeed Rev 2001; 9:19-21.

25.- Matthieu J.M, Boulat O, Bianchi N. Maternal phenylketonuria. Rev Med Suisse Romande 2001; 121:297300.

26.- Abadie V, Depondt E, Bresson JL, Vidailhet M. Dietary recommendations for pregnant women affected with phenylketonuria. Arch Pediatr 2001; 8:397-406.

27.- Koch R, Friedman E, Azen C, Hanley W, Levy H, Matalon R, et al. The International Collaborative Study of Maternal Phenylketonuria: status report. Eur J Pediatr 2000; 159 (Suppl 2):S156-60.

28.- De la Cruz F, Koch R. Genetic implications for newborn screening for phenylketonuria. Clin Perinatol 2001; 28:41924.

29.- Koch R, Hanley W, Levy H, Matalon R, Rouse B, Trefz F, et al. Maternal phenylketonuria: an international study. Mol Genet Metab 2000; 71:233-9.

30.- Sheard NF. Importance of diet in maternal phenylketonuria. Nutr Rev 2000; 58:236-9.

31.- Gazmararian JA, Solomon FM. Receipt of home health care after early discharge: results from a national. Matern Child Health J 1997; 1:151-6.

32.- Saliba Z, Bah G, Martín D, Abadie V, Azar Z, Fraisse A, et al. Pseudo-inherited form of left heart obstructive defects revealing maternal phenylketonuria. Arch Mal Coeur Vaiss 2000; 93:649-52. 